\section{Commentary: How to evaluate the effect of neoadjuvant therapy for esophageal cancer? More studies are still needed}

\author{
Haiquan Chen, MD
}

In the Chemoradiotherapy for Oesophageal Cancer Followed by Surgery Study (CROSS), pathologic complete response was observed in 28 of 121 patients with adenocarcinoma ( $23 \%$ ), and 18 of 37 with squamous-cell carcinoma $(49 \%)$, the survival benefit was more pronounced in patients with esophageal squamous cell carcinoma. ${ }^{1}$ However, the characteristics of residual tumors were not fully investigated in the CROSS study.

In a cohort of patients with esophageal squamous cell carcinoma after neoadjuvant chemoradiotherapy (nCRT), Tang and colleagues ${ }^{2}$ present the pattern of residual tumors into 4 types: I, regression toward the lumen; II, regression toward the invasive front; III, concentric regression; and IV, scattered regression. Moreover, the frequency of pathological complete response was lower in the lymph nodes $(29.2 \%)$ than in primary lesions $(33.6 \%)$. According to the pattern of tumor regression and amount of the residual tumors, $62.7 \%$ could not be detected due to potentially negative biopsy in the superficial layer. Based on pathology results, the authors investigated the characteristics of tumor regression after nCRT, offering novel information for the treatment of esophageal cancer after nCRT. However, a clinical question arises: How to predict the type of tumor regression by preoperative workup?

The relation between clinical and pathologic tumor responses is crucial in treatment decision making for patients with esophageal cancer. To evaluate the effect of neoadjuvant therapy, the Detection of Residual Disease After Neoadjuvant Chemoradiotherapy for Oesophageal Cancer

\footnotetext{
From the Department of Thoracic Surgery, Fudan University Shanghai Cancer Center, and the Institute of Thoracic Oncology, Fudan University, Shanghai, China. Disclosures: The author reported no conflicts of interest.

The Journal policy requires editors and reviewers to disclose conflicts of interest and to decline handling or reviewing manuscripts for which they may have a conflict of interest. The editors and reviewers of this article have no conflicts of interest.

Received for publication Sept 23, 2020; revisions received Sept 23, 2020; accepted for publication Sept 24, 2020; available ahead of print Oct 3, 2020.

Address for reprints: Haiquan Chen, MD, Department of Thoracic Surgery, Fudan University Shanghai Cancer Center, 270 Dong'an Rd, Shanghai 20032, China (E-mail: hqchen1@yahoo.com).

J Thorac Cardiovasc Surg 2021;162:1645-6

$0022-5223 / \$ 36.00$

Copyright (c) 2020 by The American Association for Thoracic Surgery

https://doi.org/10.1016/j.jtcvs.2020.09.109
}

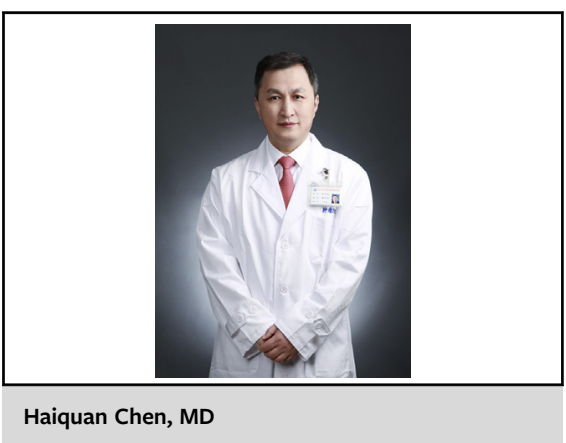

CENTRAL MESSAGE

More work is needed to increase

the accuracy of preoperative

evaluation for esophageal cancer.

The treatment decision should

be made for the best survival.

(preSANO) study was initiated to evaluate the accuracy of different diagnostic techniques for clinical response evaluations. However, with the combination of endoscopic ultrasonography, bite-on-bite biopsies, and fine-needle aspiration, 25 of 47 tumor regression grade (TRG) 1 or 2 tumors $(53.2 \%)$ and 4 of 41 TRG 3 or 4 tumors $(9.8 \%)$ were still missed. ${ }^{3}$ Thus, more work is needed to increase the accuracy of disease evaluation, particularly for TRG 1 or 2 patients.

Regarding the treatment decision after nCRT, the optimal strategy should be made for a better survival, which is the most important outcome. For this aim, the ongoing Surgery as Needed Approach in Oesophageal Cancer Patients (SANO) trial compared active surveillance with standard resection for patients who achieve a complete response after $\mathrm{nCRT}^{4}{ }^{4}$ and active surveillance could be a kind of standard care if this strategy leads to noninferior survival.

Due to the difficulties in tumor regression evaluation, on the other hand, compared with neoadjuvant therapy, postoperative adjuvant therapy was delivered based on accurate pathological staging rather than inaccurate clinical staging. In the trial comparing esophagectomies via right and left thoracic approach, which was performed under the policy of surgery plus adjuvant therapy, 5-year overall survival was $63 \%$ after esophagectomy with radical 2-field lymphadenectomy via right thoracic approach, and $51 \%$ for those with lymph node metastasis. ${ }^{5,6}$ Thus, new trials comparing neoadjuvant and adjuvant treatment for esophageal squamous cell carcinoma are needed. 
More work is needed to increase the accuracy of clinical disease staging and evaluation of tumor regression response. For patients with esophageal cancer, the optimal treatments should be established based on prospective evidence with the best outcomes.

\section{References}

1. van Hagen P, Hulshof MC, van Lanschot JJ, Steyerberg EW, van Berge Henegouwen MI, Wijnhoven BP, et al. Preoperative chemoradiotherapy for esophageal or junctional cancer. $N$ Engl J Med. 2012;366:2074-84.

2. Tang H, Jiang D, Zhang S, Zeng Z, Tan L, Hou Y, et al; Zhongshan NCRT-EC working group. Residual tumor characteristics of esophageal squamous cell carcinoma after neoadjuvant chemoradiotherapy. J Thorac Cardiovasc Surg. 2021;162: $1632-41$
3. Noordman BJ, Spaander MCW, Valkema R, Wijnhoven BPL, van Berge Henegouwen MI, Shapiro J, et al. Detection of residual disease after neoadjuvant chemoradiotherapy for oesophageal cancer (preSANO): a prospective multicentre, diagnostic cohort study. Lancet Oncol. 2018;19:965-74.

4. Noordman BJ, Wijnhoven BPL, Lagarde SM, Boonstra JJ, Coene PPLO, Dekker JWT, et al. Neoadjuvant chemoradiotherapy plus surgery versus active surveillance for oesophageal cancer: a stepped-wedge cluster randomised trial. BMC Cancer. 2018;18:142.

5. Li B, Hu H, Zhang Y, Zhang J, Miao LS, Ma LF, et al. Extended right thoracic approach compared with limited left thoracic approach for patients with middle and lower esophageal squamous cell carcinoma: three-year survival of a prospective, randomized, open-label trial. Ann Surg. 2018;267:826-32.

6. Li B, Hu H, Zhang Y, Zhang J, Sun YH, Xiang JQ, et al. Esophageal squamous cell carcinoma patients with positive lymph nodes benefit from extended radical lymphadenectomy. J Thorac Cardiovasc Surg. 2019;157:1275-83.e1. 